\title{
Is it time for quadruple therapy to be first line?
}

\author{
Loren Laine MD
}

L Laine. Is it time for quadruple therapy to be first line? Can J Gastroenterol 2003;17(Suppl B):33B-35B.

\begin{abstract}
The most commonly used regimen for Helicobacter pylori therapy at present is twice-daily proton pump inhibitor (PPI)-based triple therapy. Bismuth-based therapy is the next most common treatment used by gastroenterologists. When a PPI is combined with bismuth-based triple therapy (quadruple therapy), eradication rates are increased as compared with the triple therapy alone. Three separate randomized trials from three continents that compare quadruple therapy and PPIbased triple therapy revealed remarkably similar results. Eradication rates with PPI-based triple therapy and quadruple therapy were not significantly different. The eradication rates with quadruple therapy were $3 \%$ to $6 \%$ higher than PPI triple therapy, indicating that quadruple therapy should be no less effective than PPI triple therapy. Furthermore, these two therapies had similar rates of compliance and adverse events.

The major potential benefit of the quadruple therapy relates to antibiotic resistance. In patients with clarithromycin resistance, PPIbased triple therapy, but not quadruple therapy, had a significantly lower eradication rate. However, due to its ability to largely overcome metronidazole resistance, quadruple therapy had little if any decrement in eradication rates compared with PPI triple therapy in patients with metronidazole-resistant $H$ pylori. Therefore, quadruple therapy can be considered a first line therapy for $\mathrm{H}$ pylori.
\end{abstract}

Key Words: Bismuth; Duodenal ulcer; H pylori; Peptic ulcer

\section{Le temps de la quadrithérapie en première intention est-il arrivé?}

La forme la plus courante de traitement de l'infection à Helicobacter pylori consiste actuellement en la trithérapie fondée sur un inhibiteur de la pompe à protons (IPP) à prise biquotidienne. Vient ensuite la thérapie à base de bismuth. Lorsqu'un IPP est adjoint à la trithérapie comportant du bismuth, qui devient ainsi quadrithérapie, on observe une augmentation des taux de suppression par rapport à la trithérapie seule. Trois essais distincts, menés avec hasardisation sur trois continents, comparant la quadrithérapie à la trithérapie fondée sur un IPP ont produit des résultats remarquablement similaires. Les taux de suppression enregistrés pour la trithérapie et la quadrithérapie différaient peu. Les derniers se sont révélés de 3 à $6 \%$ supérieurs aux premiers, ce qui indique que la quadrithérapie ne devrait pas se montrer moins efficace que la trithérapie fondée sur un IPP. De plus, les chercheurs ont noté des taux similaires d'observance thérapeutique et d'effets indésirables pour les deux formes de traitement. Le grand avantage possible de la quadrithérapie a trait à la résistance aux antibiotiques. Chez les patients résistants à la clarithromycine, la trithérapie à base d'un IPP, contrairement à la quadrithérapie, a produit des taux de suppression sensiblement plus faibles. Par ailleurs, compte tenu de sa capacité de vaincre en grande partie la résistance au métronidazole, la quadrithérapie a peu ou pas été associée à une diminution des taux de suppression comparativement à la trithérapie reposant sur un IPP chez les patients porteurs d'H pylori résistant au métronidazole. Aussi peut-on envisager la quadrithérapie comme traitement de première intention de l'infection à $H$ pylori.
$\mathrm{C}$ vurrent therapy for Helicobacter pylori requires a combinatriple therapy was one of the earliest therapies used for the treatment of $\mathrm{H}$ pylori. Bismuth-based triple therapy has $H$ pylori eradication rates of approximately $80 \%$ in the United States (Product Information, Pepto-Bismol, Proctor \& Gamble, USA), with worldwide pooled eradication rates of approximately $85 \%$ (1). When a proton pump inhibitor (PPI) is combined with bismuth-based triple therapy, however, eradication rates over $95 \%$ have been achieved in some studies (2-4). This combination of PPI plus bismuth-based triple therapy has been labelled 'quadruple therapy'.

Although bismuth-based triple therapy has relatively good efficacy against $H$ pylori infection, due to its complexity (three different medications taken four times a day, commonly with the addition of an antisecretory drug), investigators sought other combinations that would be less complex, with the hope of improving compliance and, therefore, hopefully improving eradication rates. Despite early enthusiasm for dual therapies, no combination containing just two drugs can be considered acceptable therapy at this time. The most commonly used regimen for $\mathrm{H}$ pylori therapy at present is twice-daily PPI-based triple therapy. This therapy employs a PPI, clarithromycin and amoxicillin (or metronidazole) (5), and achieves an eradication rate of approximately $85 \%$ in the United States (6-8).

An additional factor that needs to be considered in choosing an $\mathrm{H}$ pylori therapy is antibiotic resistance. The two antibiotics to which $H$ pylori has clinically significant rates of baseline resistance (pretreatment) are metronidazole and clarithromycin. These drugs are important components of $\mathrm{H}$ pylori therapeutic regimens (eg, PPI-based triple therapy, bismuthbased therapies). In the United States, the baseline resistance to metronidazole is $26 \%$ (by agar dilution) and the resistance rate for clarithromycin is $10 \%(9)$. After treatment with either of these agents, it must be assumed that a majority of patients with unsuccessful therapy have emergence of $H$ pylori strains resistant to the antibiotic.

Although in vitro metronidazole resistance commonly decreases eradication rates with most metronidazole-containing regimens, these patients still derive benefit with metronidazole

This article was originally presented at a conference entitled "Helicobacter pylori: Basic Mechanisms to Clinical Cure 2002", sponsored by Axcan Pharma, November 10-14, 2002, Maui, Hawaii

Gastrointestinal Division, University of Southern California School of Medicine, Los Angeles, California, USA

Correspondence: Dr Loren Laine, Gastrointestinal Division, Department of Medicine, University of Southern California School of Medicine, 2025

Zonal Avenue, Los Angeles, California 90033, USA. Telephone 323-226-7995, fax 323-226-7573, e-mail llaine@usc.edu 
TABLE 1

Results of randomized comparison of 14-day bismuthbased triple therapy with seven-day quadruple therapy and seven-day PPI-based triple therapy in patients with Helicobacter pylori-positive functional dyspepsia

\begin{tabular}{lccc}
\hline & BMT-14 & PBMT-7 & PAC-7 \\
\hline Eradication (ITT) & $95 / 137(69 \%)^{*}$ & $110 / 134(82 \%)$ & $104 / 134(78 \%)$ \\
Eradication (PP) & $75 / 101(74 \%)^{*}$ & $92 / 105(88 \%)$ & $94 / 114(82 \%)$ \\
Eradication (ITT): & $16 / 21(76 \%)$ & $16 / 20(80 \%)$ & $17 / 23(74 \%)$ \\
$\quad$ metronidazole-sensitive & & & \\
$\quad$ Eradication (ITT): & $16 / 29(55 \%)^{* *}$ & $17 / 21(81 \%)$ & $20 / 23(87 \%)$ \\
$\quad$ metronidazole-resistant & & & \\
Compliance less than 90\% & $15 \% \%^{*, * *}$ & $6 \%$ & $3 \%$ \\
Moderate to severe AE & $45 \%^{*, * *}$ & $25 \%$ & $23 \%$ \\
AE leading to discontinuation & $9 \% * *$ & $3 \%$ & $2 \%$ \\
\hline
\end{tabular}

Data from Katelaris et al (12). ${ }^{*} P<0.05$ versus $P B M T-7 ;{ }^{* *} P<0.05$ versus PAC-7. AE Adverse events; BMT-14 Biskalcitrate, metronidazole, tetracycline; ITT Intent to treat; PAC-7 Pantoprazole, amoxicillin, clarithromycin; PBMT-7 Pantoprazole, bismuth subcitrate, metronidazole, tetracycline; PP Per protocol; PPI Proton pump inhibitor

therapy. A number of strategies have been reported to potentially help overcome metronidazole resistance, including cotherapy with a PPI or bismuth, longer duration of therapy, higher dose of metronidazole, and use of tetracycline instead of amoxicillin as part of bismuth-based therapy $(10,11)$.

In contrast, no strategy has been shown to aid in overcoming clarithromycin resistance, and clarithromycin adds very little to therapeutic regimens in patients with in vitro clarithromycin resistance. A systematic review indicated cure rates of $0 \%$ to $48 \%$ for clarithromycin-resistant $H$ pylori in trials of a PPI, clarithromycin and amoxicillin or metronidazole (1). Large American trials of a PPI, amoxicillin and clarithromycin triple therapy revealed eradication rates of $27 \%$ (6) and $45 \%$ (8) in patients with clarithromycin-resistant $\mathrm{H}$ pylori.

Thus, both PPI-based triple therapy and bismuth-based therapy appear to have good rates of eradication, and they are the two types of regimens most widely used by gastroenterologists (5). The present review will explore randomized comparisons between PPI-based triple therapy and bismuth-based therapy with attention to their eradication rates, efficacy related to antibiotic resistance, compliance and side effects.

\section{RANDOMIZED TRIALS OF QUADRUPLE THERAPY AND PPI-BASED TRIPLE THERAPY}

Katelaris et al (12) performed a multicentre randomized comparison of quadruple therapy for seven days (PBMT-7: pantoprazole $40 \mathrm{mg}$ twice daily, bismuth subcitrate $108 \mathrm{mg}$ four times daily, metronidazole $200 \mathrm{mg}$ three times daily, tetracycline $500 \mathrm{mg}$ four times daily), bismuth triple therapy for 14 days (BMT-14), and PPI triple therapy for seven days (PAC-7: pantoprazole $40 \mathrm{mg}$ twice daily, amoxicillin $1 \mathrm{~g}$ twice daily, clarithromycin $500 \mathrm{mg}$ twice daily) in 405 Australian patients with endoscopy-negative dyspepsia. The results are shown in Table 1. The eradication rates of the quadruple therapy and PPI triple therapy were not significantly different but were better than the bismuth-based triple therapy. Compliance and moderate to severe adverse events (including events leading to discontinuation) were also similar for the quadruple therapy and the PPI-based triple therapy, while the bismuth-based triple therapy fared significantly worse in these categories. Eradication rates with the quadruple therapy were virtually
TABLE 2

Results of North American randomized trial of 10-day courses of single-triple capsules (biskalcitrate, metronidazole, tetracycline) plus omeprazole (OBMT) compared with PPI-based triple therapy (omeprazole, amoxicillin, clarithromycin [OAC]) in Helicobacter pyloripositive patients with duodenal ulcer diseaes

\begin{tabular}{lcc}
\hline & OBMT & OAC \\
\hline Eradication (ITT) & $121 / 138(88 \%)$ & $114 / 137(83 \%)$ \\
Eradication (PP) & $111 / 120(93 \%)$ & $108 / 124(87 \%)$ \\
Eradication (ITT): metronidazole-sensitive & $68 / 74(92 \%)$ & $60 / 71(85 \%)$ \\
Eradication (ITT): metronidazole-resistant & $41 / 451(80 \%)$ & $36 / 44(82 \%)$ \\
Eradication (ITT): clarithromycin-sensitive & $98 / 111(88 \%)$ & $93 / 101(92 \%)$ \\
Eradication (ITT): clarithromycin-resistant & $10 / 13(77 \%)$ & $3 / 14(21 \%)^{*}$ \\
Adverse events leading to discontinuation & $0 / 139(0 \%)$ & $1 / 138(1 \%)$ \\
\hline
\end{tabular}

Data from Laine et al (13). ITT Intent to treat; PP Per protocol. ${ }^{*} P<0.001$, clarithromycin-sensitive versus resistant

\begin{tabular}{|c|c|c|}
\hline \multicolumn{3}{|c|}{$\begin{array}{l}\text { TABLE } 3 \\
\text { Results of randomized trial of seven-day courses of } \\
\text { quadruple therapy (omeprazole, biskalcitrate, } \\
\text { metronidazole, tetracycline [OBMT]) compared with PPI- } \\
\text { based triple therapy (omeprazole, amoxicillin, } \\
\text { clarithromycin [OAC]) in } 339 \text { Helicobacter pylori-positive } \\
\text { patients with peptic ulcers }\end{array}$} \\
\hline & OBMT & OAC \\
\hline Eradication (ITT) & $139 / 168(83 \%)$ & $132 / 171(77 \%)$ \\
\hline Eradication (PP) & $139 / 157(89 \%)$ & $132 / 153(86 \%)$ \\
\hline Side effects & $50 / 168(30 \%)$ & $54 / 171(33 \%)$ \\
\hline (moderate to severe) & $(6 / 168[4 \%])$ & $(11 / 171[6 \%])$ \\
\hline Incomplete compliance & $12 / 168(7 \%)$ & $3 / 171(2 \%)$ \\
\hline
\end{tabular}

Data from Calvet et al (14). ITT Intent to treat; PP Per protocol

identical whether patients had metronidazole-resistant or sensitive strains.

Laine et al (13) performed a North American randomized comparison of quadruple therapy using a single-triple capsule (biskalcitrate $40 \mathrm{mg}$, metronidazole $125 \mathrm{mg}$, tetracycline $125 \mathrm{mg}$ ) three capsules four times daily (after meals and at bedtime) plus omeprazole $20 \mathrm{mg}$ twice daily (after breakfast and dinner) for 10 days (OBMT) compared with omeprazole $20 \mathrm{mg}$ twice daily, amoxicillin $1 \mathrm{~g}$ twice daily, and clarithromycin $500 \mathrm{mg}$ twice daily (before breakfast and dinner) for 10 days (OAC) in 275 patients with duodenal ulcer disease. Results are shown in Table 2. Again, results with the quadruple therapy were not significantly different from the PPI triple therapy. Furthermore, the decrease in eradication rate in patients with metronidazole-resistant $H$ pylori compared with patients with sensitive strains was relatively small and not significant $8 \%$ in the per protocol analysis and $12 \%$ in the intent-to-treat analysis). In contrast, the per prototocol eradication rate with $\mathrm{OAC}$ was markedly lower in patients with clarithromycin-resistant strains $(23 \%)$ than in patients with clarithromycin-sensitive strains $(95 \%)$. Compliance and adverse events were comparable in the two groups: only one patient withdrew due to an adverse event.

Calvet et al (14) performed a randomized comparison of seven-day courses of quadruple therapy (OBMT: omeprazole $20 \mathrm{mg}$ twice daily, bismuth subcitrate $120 \mathrm{mg}$ three times daily, metronidazole $500 \mathrm{mg}$ four times daily, tetracycline $500 \mathrm{mg}$ three times daily) with PPI triple therapy (OAC) in 339 
patients with peptic ulcers in Spain. Results are shown in Table 3. Eradication rates were not significantly different between comparable in the two treatment groups.

\section{CONCLUSIONS}

In summary, three separate randomized trials from three different continents have remarkably similar results. Eradication rates with PPI-based triple therapy and quadruple therapy were not significantly different. The eradication rates with quadruple therapy were 3\% to $6 \%$ higher than with PPI triple therapy, indicating that quadruple therapy should be no less effective than PPI triple therapy. Furthermore, these two therapies have similar rates of compliance and adverse events.

The major potential benefit of the quadruple therapy relates to antibiotic resistance. In patients with clarithromycin resistance, the quadruple therapy will have a clear advantage over the PPI triple therapy. However, due to its ability to largely overcome metronidazole resistance, quadruple therapy will produce little if any decrement in eradication rates compared with PPI triple therapy, even in patients with metronidazoleresistant $H$ pylori. Therefore, quadruple therapy can clearly be considered a first-line therapy for $\mathrm{H}$ pylori.

\section{REFERENCES}

1. Houben MHMG, van de Beek D, Hensen EF, de Craen AJM, Rauws EAJ, Tytgat GNJ. A systematic review of Helicobacter pylori eradication therapy - the impact of antimicrobial resistance on eradication rates. Aliment Pharmacol Ther 1999;13:1047-55.

2. De Boer WA, Driessen W, Jansz AR, Tytgat GN. Effect of acid suppression on efficacy of treatment for Helicobacter pylori infection. Lancet 1995;345:817-20.

3. Borody TJ, Andrews P, Fracchia G, Brandl S, Shortis NP, Bae H. Omeprazole enhances the efficacy of triple therapy in eradicating Helicobacter pylori. Gut 1995;37:477-81.

4. Hosking SW, Ling TK, Chung SC, et al. Duodenal ulcer healing by

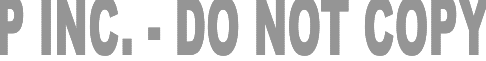

eradication of Helicobacter pylori without anti-acid treatment: randomised controlled trial. Lancet 1994;343:508-10.

5. Sharma VK, Vasudeva R, Howden CW. A survey of gastroenterologists' perceptions and practices related to Helicobacter pylori infection. Am J Gastroenterol 1999;94:3170-4.

6. Laine L, Suchower L, Frantz J, Connors A, Neil G. Twice-daily, 10day triple therapy with omeprazole, amoxicillin, and clarithromycin for $H$ pylori eradication in duodenal ulcer disease: results of 3 multicenter, double-blind U.S. trials. Am J Gastroenterol 1998;93:2106-12.

7. Fennerty MB, Kovacs TOG, Krause R, et al. A comparison of 10 and 14 days of lansoprazole triple therapy for eradication of Helicobacter pylori. Arch Intern Med 1998;158:1651-6.

8. Laine L, Fennerty MB, Osato M, et al. Esomeprazole-based Helicobacter pylori eradication therapy and the effect of antibiotic resistance: Results of 3 US multicenter, double-blind trials. Am J Gastroenterol 2000;95:3393-8.

9. Meyer JM, Silliman NP, Want W, et al. Risk factors for Helicobacter pylori resistance in the United States: the surveillance of $H$ pylori antimicrobial resistance partnership (SHARP) study, 1993-1999. Ann Intern Med 2002;136:13-24.

10. van der Wouden EJ, Thijs JC, van Zwet AA, Sluiter WJ, Kleibeuker JH. The influence of in vitro nitroimidazole resistance on the efficacy of nitroimidazole-containing anti-Helicobacter pylori regimens: a meta-analysis. Am J Gastroenterol 1999;94:1751-9.

11. van der Wouden EJ, Thijs JC, van Zwet AA, Kleibeuker JH. Review article: nitroimidazole resistance in Helicobacter pylori. Aliment Pharmacol Ther 2000;14:7-14.

12. Katelaris PH, Forbes GM, Talley NJ, Crotty B. A randomized comparison of quadruple and triple therapies for Helicobacter pylori eradication: The QUADRATE study. Gastroenterology 2002;123:1763-9.

13. Laine L, Hunt RH, El-Zimaity H, Nguyen B, Osato MS, Spenard J. Bismuth-based quadruple therapy using a single capsule of bismuth biskalcitrate, metronidazole, and tetracycline given with omeprazole versus omeprazole, amoxicillin, and clarithromycin for eradication of Helicobacter pylori in duodenal ulcer patients: A prospective, randomized, multicenter, North American trial. Am J Gastroenterol 2003;98:562-7.

14. Calvet X, Ducons J, Guardiola J, et al. One-week triple versus quadruple therapy for Helicobacter pylori infection - a randomized trial. Aliment Pharmacol Ther 2002;16:1261-7. 


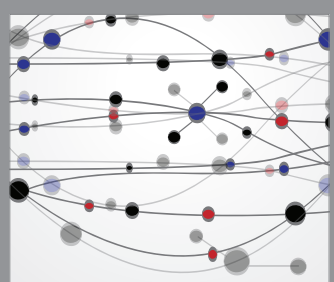

The Scientific World Journal
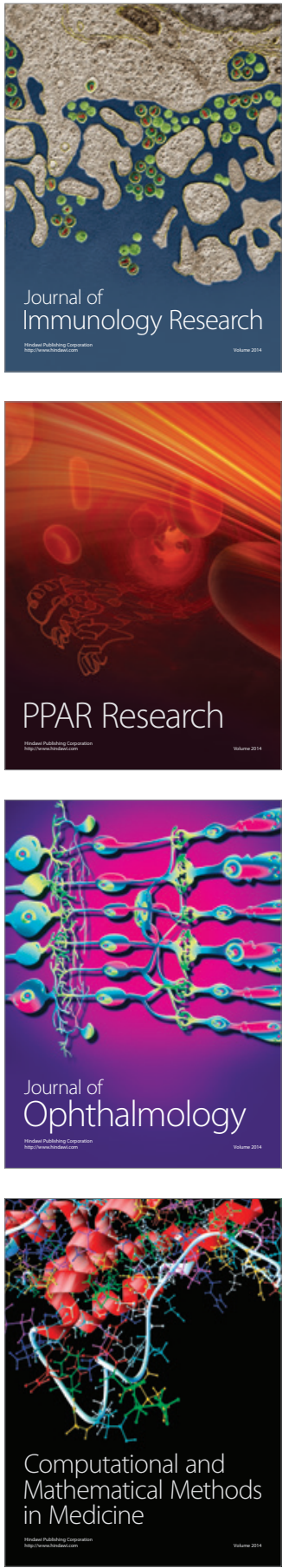

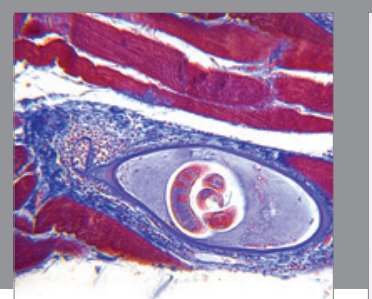

Gastroenterology Research and Practice

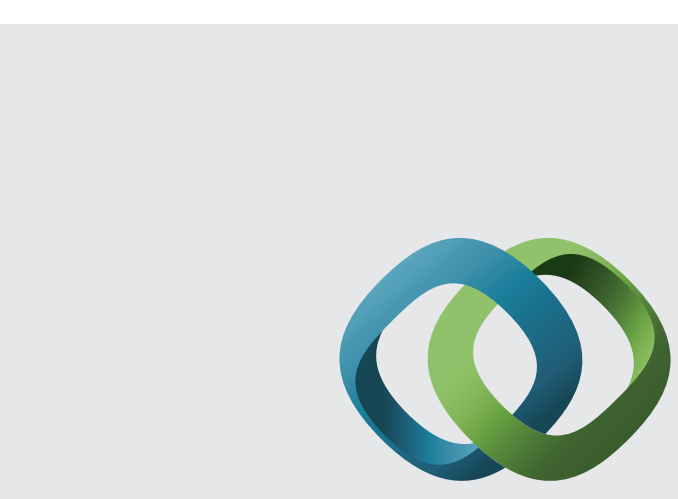

\section{Hindawi}

Submit your manuscripts at

http://www.hindawi.com
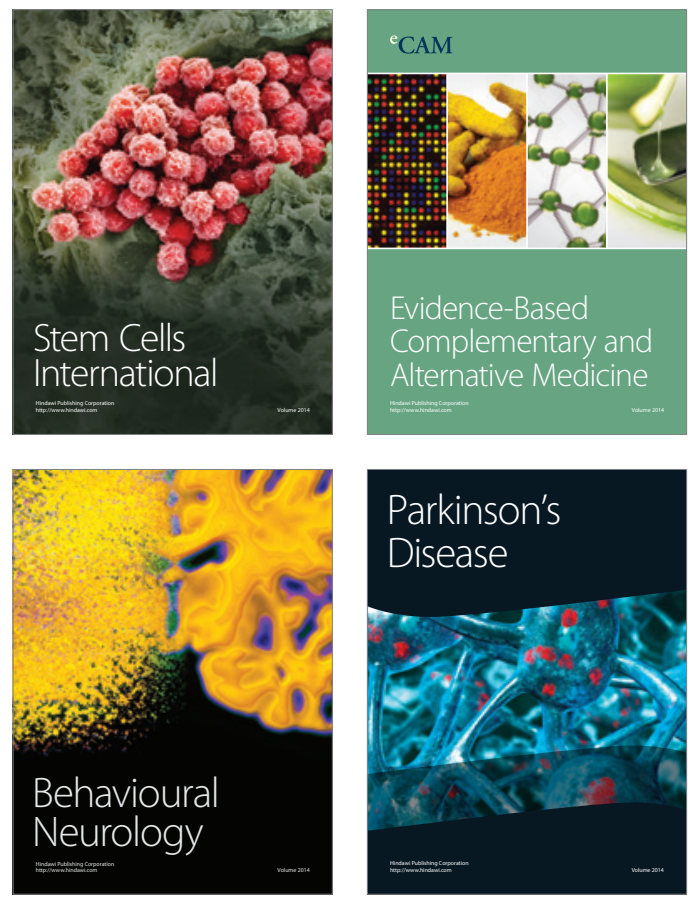
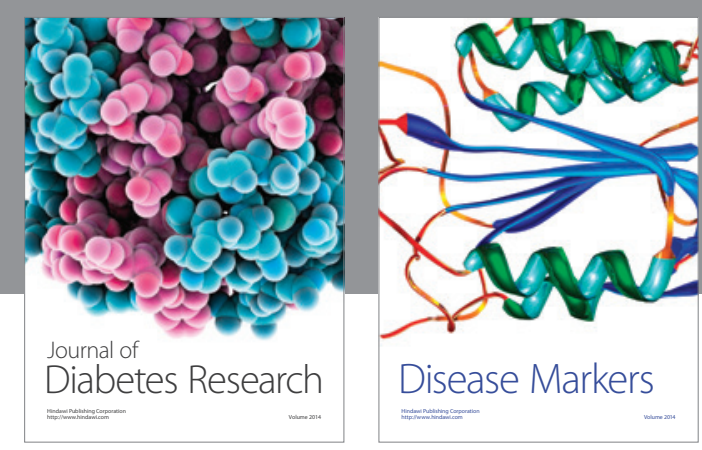

Disease Markers
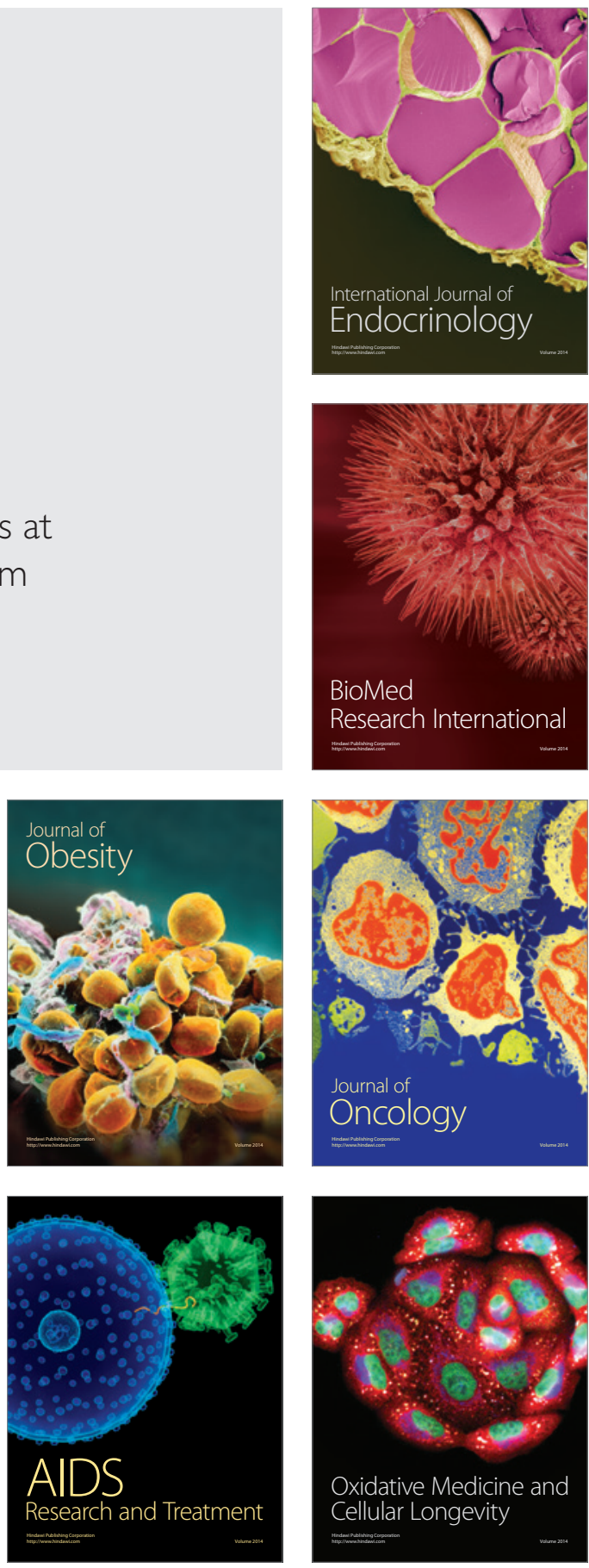